\title{
Vitamin D deficiency among postmenopausal women with low trauma acute hip fracture compared to those with fractures due to high energy trauma
}

\author{
Valizadeh M, $\mathrm{MD}^{1 *}$, Hamzehlo L, $\mathrm{MD}^{2}$, Mousavinasab N, $\mathrm{PhD}^{3}$, Amirmoghaddami HR, DCLS ${ }^{4}$, \\ Hayatbakhsh R, MD, $\mathrm{PhD}^{5}$ \\ 1- Associate Prof., Metabolic Diseases Research Center, Zanjan Faculty of Medicine, Zanjan University of Medical \\ Sciences, Zanjan, Iran. 2- Scientific Researcher, Health Centre of Zanjan University of Medical Sciences, Zanjan Faculty \\ of Medicine, Zanjan University of Medical Sciences, Zanjan, Iran. 3- Associate Prof., Dept. of Social Medicine, Faculty \\ of Medicine, Zanjan University of Medical Sciences, Zanjan, Iran. 4- Doctor of Clinical Laboratory Sciences, Dept. of \\ Clinical Laboratory, Vali E Asr General Hospital, Zanjan University of Medical Sciences, Zanjan, Iran. 5- Senior \\ Lecturer, School of Population Health, University of Queensland, Herston Road, Herston 4006, Australia.
}

\begin{abstract}
Received: November 2011, Accepted: August 2012

Background: There is no data available regarding vitamin D status among patients with hip fractures in Iran. This study aimed to determine whether postmenopausal women with low trauma hip fractures have lower vitamin D levels comparing to the control group.

Materials and Methods: Vitamin D (25-OHD) level and the prevalence of hypovitaminosis D among 73 postmenopausal women with fracture of the hip (caused by low trauma) was compared to this level among 76 controls (patients with high energy traumatic fracture of hip) in Zanjan province of Iran. Eligible patients were recruited consecutively and interviewed by trained interviewers, using a structured questionnaire. Blood samples were taken within 48 hours of the fracture and the 25-OHD level was measured.

Results: Vitamin D deficiency $(<50 \mathrm{nmol} / \mathrm{L})$ was observed in $47.7 \%$ of patients in the case group and $43.3 \%$ of women in the control group. There was no significant difference in mean 25-OHD levels and the rate of 25-OHD deficiency between the two groups.

Conclusion: Vitamin D deficiency was not more prevalent among postmenopausal women with low trauma hip fracture. The present study does not support the role of vitamin D in fragility of the hip.
\end{abstract}

Keywords: Vitamin D Deficiency, Hip Fractures, Postmenopausal

\section{Introduction}

Vitamin D is an essential factor associated with chronic health conditions, including bone health [1]. It is mainly obtained from exposure to sunlight, food and dietary supplements [2]. In conjunction with parathyroid hormone, Vitamin D serves to ensure sufficient serum calcium and phosphorus to promote optimal mineralization of the skeleton [1,3]. Vitamin $\mathrm{D}$ deficiency is a common problem in both developed and developing countries, including Iran [2, 4-8]. In a vitamin D deficiency condition bone tissue doesn't sufficiently mineralize and secondary hyperparathyroidism leads to bone resorption [3], both of which result in decreased bone strength and increased risk of the fracture [2]. It has also been found that

\footnotetext{
* Corresponding author: Majid Valizadeh, Metabolic Diseases Research Center, Zanjan University of Medical Sciences, Zanjan, Iran.

E-mail: mvalizadeh47@yahoo.com
} 
vitamin $\mathrm{D}$ deficient adults are at increased risk of falling; this is reported to be a direct result of muscle weakness caused by vitamin D deficiency [3]. Additionally, vitamin D deficiency has been found to be associated with osteoporotic fractures such as hip fractures in older adults; this has been reported to be particularly prevalent in post menopausal women [9-12].

Osteoporosis is a serious health problem in elderly people, in particular postmenopausal women. It is important to know whether vitamin $\mathrm{D}$ deficiency predicts higher rates of low trauma fractures in postmenopausal women in comparison with that among women with fractures due to high energy trauma. There has been strong evidence, mostly from the developed world, reporting that vitamin D deficiency is associated with osteoporotic hip fracture in the elderly [10, 13, 14]. For example, LeBoff et al [14] found vitamin D deficiency, defined as serum 25OHD lower than $12 \mathrm{ng} / \mathrm{mL}$, in more than half of women with osteoporotic hip fractures in the United States. There is paucity of evidence regarding the association between osteoporotic fragility and vitamin D status in developing countries. In Iran, despite a high prevalence of vitamin D deficiency, the incidence of hip fracture is low $[15,16]$.

However, there appears to be an apparent lack of empirical evidence pertaining to information on vitamin $\mathrm{D}$ levels in the Iranian women admitted with acute hip fracture. Therefore, this study aimed to identify vitamin D status in patients with osteoporotic hip fracture compared with patients who had suffered from traumatic hip fracture.

\section{Material and Methods}

This study was carried out in Zanjan, Iran. Zanjan Province is located in the northwest region of Iran, almost $300 \mathrm{~km}$ from the capital city (Tehran). Its latitude is $36.41^{\circ} \mathrm{N}$ and it has a Mediterranean climate characterized by cold snowy weather in the mountains, a moderate winter climate in the plains and moderately warm summers. Based on the national census in 2007 , the province has a population of 964601 , almost $14 \%$ of which are 50 years old or over [17].

The present study was performed at Shafiea Hospital in Zanjan. From February to September 2006, patients hospitalized with acute hip fracture were invited to participate in the study. All patients were informed of the nature and objectives of the study and written consent forms were taken from them. Patients were included in the study if they were female with at least 50 years old, having had amenorrhea for at least 12 months and were suffering from hip fracture diagnosed by a specialist and confirmed by $\mathrm{X}$-ray. Patients were excluded from the study if they had a history of consumption of drugs containing vitamin $\mathrm{D}$ or medications that influence its metabolism (during three recent months) or if they had had a fracture due to metastatic cancer.

The study participants comprised of two groups: cases and controls. Cases consisted of 73 women who were postmenopausal and were diagnosed with a fracture of the hip (caused by low trauma). The control group included 76 postmenopausal patients with hip fracture due to high energy trauma. Low trauma indicates a trauma which does not seem to generate a serious injury such as hip fracture. For example; falls from standing height or less is considered as a low trauma. High energy traumatic fracture was defined as fractures resulting from motor vehicle accidents and high height falls. Both cases and controls were selected from the patients admitted to Shafiea Hospital for hip fractures. Patients were enrolled 
consecutively and interviewed in the hospital by trained interviewers, using a structured questionnaire.

Demographic information obtained from respondents included age, marital status, employment status, living place and time and cause of fracture. Information was also collected on clinical and drug history, smoking, history of other fractures and environmental factors that could contribute to hip fracture. Participants' blood was collected during the first 48 hours of the fracture and serum was immediately frozen and stored at $-70^{\circ} \mathrm{C}$ until the laboratory test was conducted. Early collection of blood is important to rule out the effect of trauma on the level of serum 25-hydroxy vitamin D (25OHD). Serum (25-OHD) was measured by an enzyme linked immunosorbent assay (ELISA) using DRG Instruments GmbH kit. The intra- and inter-assay coefficients of variation were 13.8 and 16.2 percent, respectively. Vitamin D deficiency was defined as a 25-OHD level of less than 50 nmol/ L (20 ng per millilitre) [18, 19]. Vitamin D insufficiency was defined as a 25-
OHD level between 50 and $75 \mathrm{nmol} / \mathrm{L}$. Statistical analysis was performed using SPSS. The main characteristics of the two groups were compared using a chi-square test. As 25-OHD was not normally distributed, the Mann-Whitney U test was employed to analyze the difference between cases and controls. Categorical 25-OHD was created using different quartile levels among all patients with hip fracture and then number (and percent) of patients in each quartile was compared between the two groups. The chisquare test was also used to examine the difference in categorical 25-OHD between groups. Regression analysis was used to test the association between the patient's age and level of serum 25-OHD.

\section{Results}

Seventy three patients were diagnosed with low trauma hip fracture and 76 patients had fractures due to high energy trauma. The characteristics of the patients of both groups are shown in Table 1.

Table 1: Some characteristics of respondents in the two groups with low traumatic and high energy traumatic fracture

\begin{tabular}{lcccc}
\multicolumn{1}{c}{ Variable } & Total & Low trauma & High energy trauma fracture & P value \\
\hline Age, mean (sd) & $65.8(13.9)$ & $69.7(14.1)$ & $62.1(12.7)$ & $<.001$ \\
Vitamin D, Median & 59.1 & 53.2 & 65.6 & 0.29 \\
Living place & & & & \\
- Urban, N (\%) & $109(73.0)$ & $44(60.3)$ & $65(85.5)$ & $<.01$ \\
- Rural, N (\%) & $40(26.9)$ & $29(39.7)$ & $11(14.5)$ & \\
Previous fracture & & & $4(5.3)$ & \\
- Yes, N (\%) & $18(12.1)$ & $14(19.2)$ & $72(94.7)$ & $<.01$ \\
- No, N (\%) & $131(87.9)$ & $59(80.8)$ & & \\
Place of fracture & & & $64(84.2)$ & \\
- Indoor, N (\%) & $76(51.0)$ & $64(87.7)$ & & $<.001$ \\
- Outdoor, N (\%) & $73(49.0)$ & $9(12.3)$ & $22(28.9)$ & \\
Past medical history & $60(40.3)$ & $38(52.1)$ & $54(71.1)$ & \\
- Yes, N (\%) & $89(59.7)$ & $35(47.9)$ & & \\
- No, N (\%) & & & & \\
\hline
\end{tabular}


Patients in the fragility fracture group were older and more likely to live in rural areas $(\mathrm{p}<0.01)$. Compared with the high energy traumatic fracture group, low trauma cases reported higher rate of previous fractures, more past medical history, and were more likely to have had their fracture occur indoors $(\mathrm{p}<0.01)$. There was no statistical difference between the two groups in median of 25OHD. Serum concentrations of 25-OHD in both groups are shown in Figure 1.

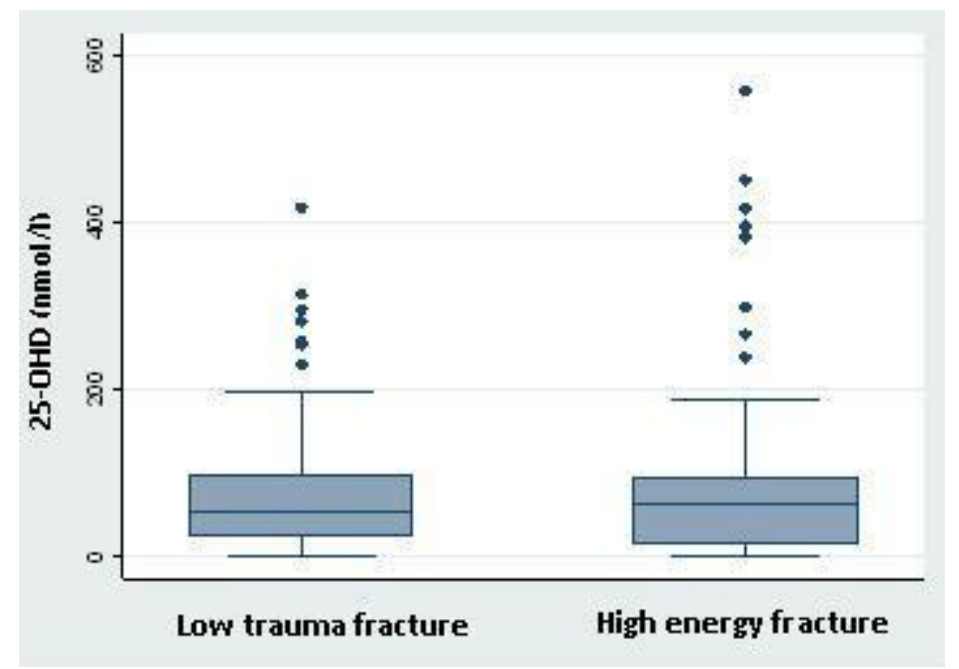

Figure 1: Serum 25-OHD $(\mathrm{nmol} / \mathrm{l})$ in patients with low trauma hip fracture (cases) and control subjects $(p=0.47)$

In a separate analysis the categorical difference in level of 25-OHD between cases and controls was tested (Table 2). In general, less than half of the participants had serum 25 -OHD level $<50 \mathrm{nmol} / \mathrm{L}$. Lowest quartile of vitamin D level (25-OHD <20.5 nmol/L) was more prevalent in the control group fracture (28.9\% versus $20.5 \%$ ), while second quartile of vitamin D level $(20.75 \mathrm{nmol} / \mathrm{L}<$ $25-\mathrm{OHD}<59.1 \mathrm{nmol} / \mathrm{L}$ ) was more frequent in the case group. We only measured serum calcium in 6 patients with vitamin D level in toxic range (> $374 \mathrm{nmol} / \mathrm{l}$ ) after vitamin $\mathrm{D}$ assay; fortunately this level was normal in all 6. However, this analysis showed that the rate of vitamin $\mathrm{D}$ deficiency and insufficiency was not significantly different between the two groups. In a separate analysis, we also examined the association between patient's age and serum 25-OHD. As the 25-OHD was not normally distributed, we $\log$ transformed the data. Regression analysis did not show a statistically significant association between age and level of vitamin D (data are not shown).

Table 2: Quartile levels of serum 25-OHD in cases and controls

\begin{tabular}{|c|c|c|c|c|c|c|}
\hline & \multicolumn{5}{|c|}{$25-\mathrm{OHD}(\mathrm{nmol} / \mathrm{L})$} & \multirow{2}{*}{$\begin{array}{r}\text { Toxic leve } \\
>374.00 \\
\end{array}$} \\
\hline & $\leq 20.75$ & $20.75-59.1$ & 59.1-98.5 & $>98.5$ & & \\
\hline Case, N (\%) & $15(20.5)$ & $25(34.2)$ & $16(21.9)$ & $17(23.3)$ & & $1(1.4)$ \\
\hline Control, N (\%) & $22(28.9)$ & $13(17.1)$ & $22(28.9)$ & $19(25)$ & 0.10 & $5(6.6)$ \\
\hline
\end{tabular}




\section{Discussion}

To the best of our knowledge, this is the first study that compares vitamin D status among Iranian post menopausal women with low trauma high energy fracture with that of patients with traumatic fracture. This study did not find a significant difference in mean vitamin $\mathrm{D}$ levels and also rate of vitamin $\mathrm{D}$ deficiency between the two groups. This is in consistent with the findings of Erem et al [20] which previously have shown that mean serum vitamin $\mathrm{D}$ of patients with osteoporotic fractures was not different with that of the normal population. Prevalence of hypovitaminosis in patients with hip fracture has been reported between $50 \%$ to $100 \%$ in different countries [21-23]. Those studies indicated that hypovitaminosis $\mathrm{D}$ is more prevalent among postmenopausal women and patients with osteoporosis (regardless of hip fracture) [21-23].

Micronutrient studies from Iran have shown that only $3.9 \%$ of postmenopausal osteoporotic women living in northwest Iran receive adequate dietary vitamin $\mathrm{D}$ [24]. This seems plausible as the people in this region have a low intake of fatty fish and their processed food is not fortified by vitamin D. However, in a study of the prevalence of hypovitaminosis $\mathrm{D}$ in different age groups in different urban areas of Iran, vitamin D deficiency in older age groups (over 50 years) is reported to be less than younger people [8, 25]. Hashemipour et al (2004) and Hesmhat and colleagues (2008) suggested parenteral vitamin $\mathrm{D}$ intake by elderly as the major differentiating factor between various age groups that could explain lower prevalence of vitamin D deficiency in elderly females. Many physicians in Iran prescribe parenteral vitamin D (containing 300,000IU vitamin D) for postmenopausal and elderly patients, particularly those with musculoskeletal complaints in short intervals (e.g., weekly) as a malpractice.

Maggio et al [26] study indicated that the risk of vitamin D deficiency and its severity increases with age especially after 70 . In a separate analysis the current data did not suggest any difference in rate of vitamin D deficiency among cases older than 70 years (data are not shown). In addition, findings of the present study do not agree with previous research that reported a higher rate of vitamin $\mathrm{D}$ deficiency among residents of urban areas or lower socioeconomic status [27, 28].

The present study must be interpreted with cautious as there were important limitations to note. First, serum PTH was not measured due to limited resources and future research may endeavour to address this limitation as an assessment of serum PTH in relation to vitamin $\mathrm{D}$ could indicate the presence of secondary hyperparathyroidism that can lead to cortical bone loss and increase risk of fracture. Second, bone densitometry was not measured in this study and the patients were categorised according to the severity of trauma. This may have caused misclassification of the patient, i.e. some of the patients categorised as control may have had osteoporosis as well. Third, we selected the control group from hospital patients, rather than normal population. This may have led to an absence of any significant difference in vitamin D levels between cases and controls. However, mean and median serum vitamin D levels in this study are higher than the community-dwelling women studied by Hashemipour and colleagues [8]. Finally, there was no data about the duration of sun-exposure in the participants. Although sun-exposure is considered an important factor in vitamin $\mathrm{D}$ deficiency, previous study in Iran has shown that exposure to sunlight is not associated with serum levels of vitamin D [8]. 


\section{Conclusion}

The present study indicates a lower rate of vitamin D deficiency among patients with acute hip fracture compared to similar studies. The present data do not suggest an association between vitamin D deficiency and low trauma fracture. Despite a high prevalence of vitamin D deficiency in the young population of Iran, minority of elderly women were detected to have lowest quartile of vitamin D level. This may justify a low incidence of hip fractures among post menopausal women in Iran.

\section{Acknowledgments}

The authors would like to thank research nurse Mrs. Misaghi, for patient recruitment and assistance in data collection and also all patients who participated in this project. This research was supported by Zanjan University vice-chancellor in research affairs as an approved student project.

\section{Conflict of interest: Non declared}

\section{References}

1. Holick MF. Sunlight and vitamin D for bone health and prevention of autoimmune diseases, cancers, and cardiovascular disease. Am J Clin Nutr 2004; 80(6Suppl):1678S-88S.

2. Holick MF. Vitamin D deficiency. N Engl J Med 2007; 357(3):266-81.

3. DeLuca HF. Overview of general physiologic features and functions of vitamin D. Am J Clin Nutr 2004; 80(6 Suppl):1689S-96S.

4. Erkal MZ, Wilde J, Bilgin Y, Akinci A, Demir E, Bodeker RH \& et.al. High prevalence of vitamin D deficiency, secondary hyperparathyroidism and generalized bone pain in Turkish immigrants in Germany: identification of risk factors. Osteoporos Int 2006; 17(8):1133-40.
5. Holick MF, Siris ES, Binkley N, Beard MK, Khan A, Katzer JT \& et.al. Prevalence of Vitamin D inadequacy among postmenopausal North American women receiving osteoporosis therapy. J Clin Endocrinol Metab 2005; 90(6):3215-24.

6. Alagol F, Shihadeh Y, Boztepe H, Tanakol R, Yarman S, Azizlerli H \& et.al. Sunlight exposure and vitamin $\mathrm{D}$ deficiency in Turkish women. $\mathbf{J}$ Endocrinol Invest 2000; 23(3):173-7.

7. Goswami R, Gupta N, Goswami D, Marwaha RK, Tandon N, Kochupillai N. Prevalence and significance of low 25-hydroxyvitamin D concentrations in healthy subjects in Delhi. Am J Clin Nutr 2000; 72(2):472-5.

8. Hashemipour S, Larijani B, Adibi H, Javadi E, Sedaghat M, Pajouhi M \& et.al. Vitamin D deficiency and causative factors in the population of Tehran. BMC Public Health 2004; 4:38.

9. Bakhtiyarova S, Lesnyak O, Kyznesova N, Blankenstein MA, Lips P. Vitamin D status among patients with hip fracture and elderly control subjects in Yekaterinburg, Russia. Osteoporos Int 2006; 17(3):441-6.

10. Nuti R, Martini G, Valenti R, Gambera D, Gennari L, Salvadori S \& et.al. Vitamin D status and bone turnover in women with acute hip fracture. Clin Orthop Relat Res 2004; (422):208-13.

11. Sakuma M, Endo N, Oinuma T, Hayami T, Endo E, Yazawa T \& et.al. Vitamin D and intact PTH status in patients with hip fracture. Osteoporos Int 2006; 17(11):1608-14.

12. Thiebaud D, Burckhardt P, Costanza M, Sloutskis D, Gilliard D, Quinodoz F \& et.al. Importance of albumin, 25(OH)-vitamin D and IGFBP-3 as risk factors in elderly women and men with hip fracture. Osteoporos Int 1997; 7(5):457-62.

13. Cumming RG, Klineberg RJ. Case-control study of risk factors for hip fractures in the elderly. Am J Epidemiol 1994; 139(5):493-503.

14. LeBoff MS, Kohlmeier L, Hurwitz S, Franklin J, Wright J, Glowacki J. Occult vitamin D deficiency in postmenopausal US women with acute hip fracture. JAMA 1999; 281(16):1505-11.

15. Moayyeri A, Soltani A, Larijani B, Naghavi M, Alaeddini F, Abolhassani F. Epidemiology of hip fracture in Iran: results from the Iranian Multicenter Study on Accidental Injuries. Osteoporos Int 2006; 17(8):1252-7.

16. Valizadeh M, Mazloomzadeh S, Azizi R. Epidemiology of hip fracture in Zanjan, Iran. Arch Osteoporos 2008; 3:1-5.

17. General population and housing census in Iran. 2007. Statistical Center of Iran . Available at: URL: http://www.Amar.org.ir/. Accessed 2007. 
18. Malabanan A, Veronikis IE, Holick MF. Redefining vitamin D insufficiency. Lancet 1998; 351(9105):805-6.

19. Bischoff-Ferrari HA, Giovannucci E, Willett WC, Dietrich T, Dawson-Hughes B. Estimation of optimal serum concentrations of 25hydroxyvitamin D for multiple health outcomes. Am J Clin Nutr 2006; 84(1):18-28.

20. Erem C, Tanakol R, Alagol F, Omer B, Cetin O. Relationship of bone turnover parameters, endogenous hormones and vit D deficiency to hip fracture in elderly postmenopausal women. Int $\mathbf{J}$ Clin Pract 2002; 56(5):333-7.

21. Beringer T, Heyburn G, Finch M, McNally C, McQuilken M, Duncan M \& et.al. Prevalence of vitamin D inadequacy in Belfast following fragility fracture. Curr Med Res Opin 2006; 22(1):101-5.

22. Brown IR, Bakowska A, Millard PH. Vitamin D status of patients with femoral neck fractures. Age Ageing 1976; 5(3):127-31.

23. Gaugris S, Heaney RP, Boonen S, Kurth H, Bentkover JD, Sen SS. Vitamin D inadequacy among post-menopausal women: a systematic review. QJM 2005; 98(9):667-76.

24. Hejazi J, Mohtadinia J, Kolahi S, EbrahimiMamaghani M. Nutritional status among postmenopausal osteoporotic women in North West of Iran. Asia Pac J Clin Nutr 2009; 18(1):48-53.

25. Heshmat R, Mohammad K, Majdzadeh SR, Forouzanfar MH, Bahrami A, Ranjbar GH \& et.al. Vitamin D deficiency in Iran: A multi-center study among different urban areas. Iranian J Publ Health 2008; A supplementary issue on Osteoporosis and Bone Turnover(1):72-8.

26. Maggio D, Cherubini A, Lauretani F, Russo RC, Bartali B, Pierandrei M \& et.al. 25(OH)D Serum levels decline with age earlier in women than in men and less efficiently prevent compensatory hyperparathyroidism in older adults. J Gerontol A Biol Sci Med Sci 2005; 60(11):1414-9.

27. Islam MZ, Lamberg-Allardt C, Karkkainen M, Outila T, Salamatullah Q, Shamim AA. Vitamin D deficiency: a concern in premenopausal Bangladeshi women of two socio-economic groups in rural and urban region. Eur J Clin Nutr 2002; 56(1):51-6.

28. Lips P. Vitamin D deficiency and osteoporosis: the role of vitamin $\mathrm{D}$ deficiency and treatment with vitamin $\mathrm{D}$ and analogues in the prevention of osteoporosis-related fractures. Eur J Clin Invest 1996; 26(6):436-42. 